\title{
Dynamics of bootstrap percolation
}

\author{
Prabodh Shukla* \\ Physics Department \\ North Eastern Hill University \\ Shillong-793 022, India
}

\begin{abstract}
Bootstrap percolation transition may be first order or second order, or it may have a mixed character where a first order drop in the order parameter is preceded by critical fluctuations. Recent studies have indicated that the mixed transition is characterized by power law avalanches, while the continuous transition is characterized by truncated avalanches in a related sequential bootstrap process. We explain this behavior on the basis of a through analytical and numerical study of the avalanche distributions on a Bethe lattice.
\end{abstract}

\section{INTRODUCTION}

Bootstrap percolation was introduced in 1979 [1] to study the diminution and eventual destruction of magnetic order by non-magnetic impurities in a magnet. As impurity increases, not only the concentration of magnetic ions decreases but also an increasing number of magnetic ions find themselves surrounded by insufficient number of magnetic neighbors to retain their magnetism. This effect accelerates the destruction of magnetic order, and sometimes changes a continuous second order transition as seen in ordinary percolation [2] to a discontinuous first order transition. The first order transition encountered in bootstrap problems has often a mixed character in the sense that the discontinuous drop in magnetization is preceded by critical fluctuations characteristic of a second order transition. The precise criterion for the occurrence of a mixed transition is not very clear, and has been the subject of research in recent years [3, 4, 5, 6]. The investigations have relied upon numerical simulations in the case of regular d-dimensional lattices, and analytic solutions in the case of a Bethe lattice. In some borderline cases, the presence of critical fluctuations at the threshold of a discontinuity makes it difficult to distinguish numerically between a true discontinuity, and a steep but continuous decrease in the order parameter. A recent study of the distribution of avalanches in the bootstrap dynamics has suggested a convenient criterion for distinguishing between the two cases [5]. The suggestion is that the mixed transitions are characterized by power-law avalanches while second order transitions are characterized by truncated avalanches. The purpose of this article is to expand on this point by presenting a through analytic and numerical study of the problem on a Bethe lattice. The Bethe lattice does not capture all the complexities of bootstrap dynamics on periodic lattices but it does provide useful insight into what makes the avalanche distributions different in the two cases. The following presentation is self-contained and does not assume any prior knowledge of bootstrap percolation on the part of an interested reader. However, before we begin it may be appropriate to mention that in recent years bootstrap percolation has grown beyond the field of disordered magnetism, and found several new applications in physics, materials science, biology, electronic communication, and social networks. It has also acquired a new name and is sometimes called k-core percolation. We shall not go into these aspects here, but an interested reader will find useful links to these problems in reference [5].

Consider a lattice whose sites are either occupied with probability $p$ or empty with probability $1-p$. The empty sites need not be devoid of matter but rather they may be occupied by a different species of atoms that are not relevant for our discussion. The relevant interactions are among pairs of nearest neighbor occupied sites. In the limit $p \rightarrow 0$, the occupied sites are isolated and can not exhibit cooperative effects. On the other hand, they exhibit strong cooperative effects in the limit $p \rightarrow 1$. Intuitively, we expect the transition from independent to cooperative behavior at a threshold $p=p_{c}$ when the occupied sites begin to form a connected cluster spanning the entire lattice. This is known as the percolation point, and $p_{c}$ the percolation probability [2]. The usual approach of percolation theory is to start with a lattice with a fraction $p$ of its sites occupied randomly, and search for the smallest value of $p$ where the occupied sites contain a spanning cluster with probability unity. Bootstrap percolation [1] asks a slightly more general question. Given a lattice (of coordination number $z$ ) whose sites are randomly occupied with probability $p$, what is the smallest value of $p$ at which the lattice contains a spanning cluster of occupied sites such that each site in the cluster has at least $m(0 \leq m \leq z)$ nearest neighbors. The usual approach to this problem is again to start with a lattice with a fraction $p$ of its sites occupied randomly, and recursively cull all sites that have less than

*Electronic address: shukla@nehu.ac.in 
$m$ neighbors. The smallest value of $p$ for which a spanning cluster survives the culling process is the $m$-bootstrap percolation probability. The bootstrap percolation probability for $m=2$ is identical with the ordinary percolation probability. Thus the $m$-bootstrap process is a generalization of the ordinary percolation process. In the following, we shall use the word percolation to mean bootstrap percolation for an arbitrary value of $m$ unless specified otherwise.

In order to determine $p_{c}$ numerically, one has to examine a randomly occupied lattice over a range of values of $p$. For each $p$, a configuration of occupied sites is generated. Then sites with less than $m$ nearest neighbors are culled recursively. This procedure either empties the lattice, or yields a stable configuration (called an $m$-core) in which every occupied site has at least $m$ occupied nearest neighbors. The data is averaged over several configurations for each value of $p$. The smallest value of $p$ at which the probability of finding an $m$-core is unity is identified with the percolation probability $p_{c}$. We call this a one-shot bootstrap procedure to emphasize that the initial configuration is subjected to bootstrap culling only once, and no stable site is culled in this procedure.

We now describe a different bootstrap process $[3,44,5]$ that we call the sequential bootstrap process. It comprises the following steps:

Step-1: Start with a fully occupied lattice.

Step-2: Attack an occupied site randomly i.e. remove it.

Step-3: Remove all unstable sites recursively, and count the total number of sites removed including the stable site removed in step-2.

Step-4: If the lattice is not empty, go to step-2 and repeat.

The result of the above exercise is a list of integers $\left\{a_{1}, a_{2}, a_{3}, \ldots\right\}$; where $a_{k}$ is the size of the $k$-th avalanche. Some interesting questions are: (i) how many attacks does it take to empty the lattice? If it takes kmax attacks to empty a lattice of $N$ sites, the quantity of thermodynamic interest is the ratio $k m a x / N$, (ii) what is the distribution of the avalanches $\left\{a_{k}\right\}$ ? (iii) the fractional occupation of the stable lattice $\left(p_{c}\right)$ before the last attack, (iv) the size of the last avalanche $a_{k \max }$ that empties the lattice, and (v) the nature of fluctuations in the system just before the last avalanche. The reason for the interest in the size of the last avalanche is the following. If the last avalanche is microscopic, the transition at $p=p_{c}$ is second order. If the last avalanche is macroscopic, the transition is first order. Second order phase transitions exhibit critical fluctuations in their vicinity, and we find this to be the case here as well. Normally, first order transitions are not associated with critical fluctuations. However, in the present case, we find power-law (critical) fluctuations at the threshold of the first order transition $\left(p \rightarrow p_{c}^{+}\right)$. In this respect, the bootstrap process is interesting. It provides a caricature of fluctuation driven first-order phase transitions.

Although it is not immediately obvious, the sequential bootstrap process described above has a close relationship with the one-shot bootstrap. Indeed, the results of the one-shot bootstrap can be obtained from those of the sequential bootstrap. Thus the sequential bootstrap is a more general bootstrap process, and includes one-shot bootstrap as a special case. We shall return to this point after we have established some results concerning the one-shot process on a Cayley tree.

\section{BOOTSTRAP DYNAMICS ON A CAYLEY TREE}

An exact solution of the one-shot bootstrap dynamics on the Bethe lattice has been obtained [5] following the method developed in the context of the zero-temperature dynamics of random field Ising model [7, 8]. It is useful to look at this analysis in somewhat more detail. We take a random configuration, and put culling tags on sites that have less than $m$ nearest neighbors. When a site is culled, it does not affect other culling tags. This means that the culling process is Abelian, and the final result does not depend on the order in which we cull unstable sites. We take advantage of this Abelian property, and initiate the culling process from the surface of the Cayley tree. Let the surface be $n+1$ steps away from the root of the tree, and $P^{n}(z, m, p)$ be the conditional probability that a site $n$ steps away from the root is not culled given that it has a nearest neighbor at a distance of $n-1$ steps from the root. In the following, the arguments in $P^{n}(z, m, p)$ will be omitted if doing so does not cause any confusion. Thus, $P^{n}$ is determined by the recursion relation,

$$
\begin{array}{r}
P^{n}=p \sum_{k=0}^{z-1}\left(\begin{array}{c}
z-1 \\
k
\end{array}\right)\left[P^{n+1}\right]^{k}\left[1-P^{n+1}\right]^{z-1-k} p_{k+1}, \\
p_{k+1}=1 \text { if } k+1 \geq m, \\
p_{k+1}=0 \text { if } k+1<m .
\end{array}
$$

The rationale for the above equation is as follows. A site at a distance of $n$ steps from the root has one nearest neighbor at level $n-1$ and $z-1$ nearest neighbors at level $n+1$. The neighbors at level $n+1$ are independent of each other in deciding whether to cull the site at level $n$. We iterate equation (1) starting at the boundary of a large 
tree with $P^{n+1}=p$, and work inward. The equation iterates to a fixed point solution $P^{*}$ in the limit $n \rightarrow \infty ; P^{*}$ is the conditional probability that deep inside the tree, a nearest neighbor of the root is occupied given that the root is occupied. The unconditional probability that the root is occupied is given by,

$$
P=p \sum_{k=0}^{z}\left(\begin{array}{l}
z \\
k
\end{array}\right)\left[P^{*}\right]^{k}\left[1-P^{*}\right]^{z-k} p_{k}
$$

If the root is occupied it must lie on an unbroken network of occupied sites going all the way to the boundary of the tree. Thus $P$ is also the $m$-bootstrap percolation probability, i.e. the probability that a site in the deep interior of the tree is a part of an infinite $m$-cluster. The equations apply to the deep interior of the tree because a stable fixed point of the recursion relation is insensitive to the boundary of the tree. The deep interior part of the Cayley tree is also known as the Bethe lattice, or a random graph of a fixed coordination number $z$. Equation (2) was also obtained[1] from a mean field argument that happens to be exact for the bootstrap problem on a Bethe lattice. Our approach emphasizes the Abelian property of the bootstrap dynamics and provides a rigorous justification for the mean field equations in this case. This is important because it is in general not possible to justify the mean field equations even on a Bethe lattice beyond the statement that they are self-consistent.

In the case $m=2$, i.e. $p_{0}=p_{1}=0$, and $p_{2}=p_{3}=\ldots=p_{z}=1$, equations (1) and (2) reduce to the well known equations for ordinary percolation on a Bethe lattice [2]:

$$
\begin{array}{r}
P^{*}=p\left[1-\left(1-P^{*}\right)^{z-1}\right], \\
P=p\left[1-\left(1-P^{*}\right)^{z}-z P^{*}\left(1-P^{*}\right)^{z-1}\right] \quad(m=2)
\end{array}
$$

The ordinary percolation $(m=2)$ is a second order transition. The percolation probability is given by $p_{c}=\frac{1}{(z-1)}$; the order parameter $P=0$ if $p \leq p_{c}$, and increases continuously with $p$ for $p>p_{c}$.

For $m \geq 3$, the percolation transition on the Bethe lattice is a first order transition; $p_{c}$ as well as the jump in the order parameter $P$ at $p=p_{c}$ increases steadily with increasing values of $m$. For $m=z\left(p_{0}=p_{1}=\ldots=p_{z-1}=0 ; p_{z}=1\right)$, $P^{*}$ is determined by the equation $P^{*}=p\left[P^{*}\right]^{z-1}$. The possible solutions are $P^{*}=0$ and $P^{*}=p^{-\frac{1}{z-2}}$. But physically acceptable solutions are: $P^{*}=0$ for $p<1$, and $P^{*}=P=1$ for $p=1$, indicating a first order transition at $p_{c}=1$.

The case $m=z-1\left(p_{0}=p_{1}=\ldots=p_{z-2}=0 ; p_{z-1}=p_{z}=1\right)$ yields pleasing closed form expressions for $p_{c}$ and the discontinuity in the order parameter at $p=p_{c}$. In this case, $P^{*}$ is determined by the equation:

$$
P^{*}=p\left[(z-1)\left(P^{*}\right)^{z-2}\left(1-P^{*}\right)+\left(P^{*}\right)^{z-1}\right] \quad(m=z-1)
$$

Obviously $P^{*}=0$ is a solution of equation (4). Other non-zero solutions of equation (4) are given by the equation,

$$
f\left(p, P^{*}\right)=p\left[(z-1)\left(P^{*}\right)^{z-3}\left(1-P^{*}\right)+\left(P^{*}\right)^{z-2}\right]-1=0 \quad(m=z-1)
$$

In the range $p_{c}<p \leq 1$, equation (5) has two real solutions that merge at $p=p_{c}$ ( $p_{c}$ to be calculated below), and vanish from the real axis for $p<p_{c}$. $P^{*}$ makes a discontinuous jump from $P^{*}=P_{\text {disc }}^{*}$ to $P^{*}=0$ as $p$ crosses the value $p=p_{c}$ from above. The non-zero solution for $P^{*}$ at the double root is determined by the equation,

$$
\left.\frac{d f\left(p_{c}, P^{*}\right)}{d P^{*}}\right|_{P^{*}=P_{d i s c}^{*}}=0 \Longrightarrow P_{d i s c}^{*}=\frac{(z-1)(z-3)}{(z-2)^{2}} \quad(m=z-1)
$$

Substituting from equation (6) into equation (5) gives,

$$
p_{c}=\left(\frac{z-3}{z-2}\right)\left[\frac{(z-2)^{2}}{(z-1)(z-3)}\right]^{z-2} \quad(m=z-1)
$$

\section{POWER-LAW AVALANCHES}

We now show that there are critical fluctuations in the size of the avalanche as $p \rightarrow p_{c}^{+}$, irrespective of whether there is a first order or a second order transition at $p=p_{c}$. Let us focus on a pair of nearest neighbor occupied sites in a stable $m$-core. Designate one of the occupied sites as the root of the tree. The other site lies at the vertex of a sub-tree connected to the root. Now remove the root by hand. Let $\pi_{a}$ be the probability that this causes an avalanche of size- $a$ on the sub-tree. For example,

$$
\pi_{0}=p \sum_{k=0}^{z-1}\left(\begin{array}{c}
z-1 \\
k
\end{array}\right)\left[P^{*}\right]^{k}\left[1-P^{*}\right]^{z-1-k} p_{k} .
$$


In general, it is convenient to work with a normalized generating function $\pi(x)$,

$$
\pi(x)=\sum_{a=0}^{\infty} \pi_{a} x^{a} ; \quad\left[\pi(x=1)=P^{*}\right] .
$$

The generating function is determined by the equation,

$$
\pi(x)=\pi_{0}+x p \sum_{k=0}^{z-1}\left(\begin{array}{c}
z-1 \\
k
\end{array}\right)[\pi(x)]^{k}\left[1-P^{*}\right]^{z-1-k}\left[p_{k+1}-p_{k}\right]
$$

When we remove the root, avalanches may be initiated on any one of the $z$ subtrees connected to the root if the vertex of that sub-tree is occupied. Let $\Pi_{a}$ be the probability that a total avalanche of size- $a$ is initiated by the removal of the root. The generating function for $\Pi_{a}$ and its equation is,

$$
\begin{array}{r}
\Pi(x)=\sum_{a=0}^{\infty} \Pi_{a} x^{a} ; \quad \Pi_{a}=\left.\frac{1}{a !} \frac{d^{a} \Pi(x)}{d x^{a}}\right|_{x=0} . \\
\Pi(x)=x p \sum_{k=0}^{z}\left(\begin{array}{l}
z \\
k
\end{array}\right)[\pi(x)]^{k}\left[1-P^{*}\right]^{z-k} I(z, m, k)
\end{array}
$$

The last factor $I(z, m, k)$ in the above equation takes care of the notion of "removing the root by hand". It is the probability that a stable root with $k$ occupied nearest neighbors would become unstable if $p$ were to decrease by an infinitesimal amount from $p$ to $p-\delta p$.

We are primarily interested in the behavior of $\Pi_{a}$ in the limit $a \rightarrow \infty$. This is determined by $\Pi(x)$ in the limit $x \rightarrow 1$. We calculate $\Pi(x \rightarrow 1)$ for $z=4$, and $m=3$. The algebra is relatively simple in this case but the main result of the analysis holds for all $z \geq 4$ and $m \geq 3$. We note that $P^{*}(4,3, p)$ is either zero, or it is determined by the quadratic equation,

$$
2 p\left[P^{*}\right]^{2}-3 p P^{*}+1=0 ; \quad P^{*}=\frac{3 p \pm \sqrt{9 p^{2}-8 p}}{4 p} \quad(z=4, m=3)
$$

The two non-zero solutions are real in the range $\frac{8}{9} \leq p \leq 1$, and merge into a double root $P_{d i s c}^{*}=\frac{3}{4}$ at $p_{c}=\frac{8}{9}$. For $p<p_{c}$, the solutions of the quadratic equation disappear from the real plane, and we are left with only one solution $P^{*}=0$ that is physically acceptable. Thus $P^{*}$ jumps from the value $P^{*}(4,3, p)=\frac{3}{4}$ to $P^{*}(4,3, p)=0$, as $p$ crosses the value $p_{c}=\frac{8}{9}$ from above. The order parameter $P(p)$ jumps from $P(p)=\frac{21}{32} \approx .65$ to $P(p)=0$. For $p>p_{c}$, we choose the upper sign of the square root. Writing $p=p_{c}+\delta p$, equation (12) gives to the leading order in $\delta p$,

$$
P^{*}\left(p_{c}+\delta p\right)=P_{d i s c}^{*}+\frac{9}{8 \sqrt{2}}(\delta p)^{\frac{1}{2}} \quad(z=4, m=3)
$$

For $z=4, m=3$, equation (10) for $\pi(x)$ takes the quadratic form,

$$
3 x p\left[1-P^{*}\right] \pi^{2}(x)-\pi(x)+p\left[P^{*}\right]^{3}=0 \quad(z=4, m=3)
$$

Although equation (14) can be easily solved, it is instructive to examine it in the vicinity of $\pi(x=1)=P^{*}$. Let $x=1-\delta x$, and $\pi(x=1-\delta x)=P^{*}+\delta \pi$, where $\delta x$, and $\delta \pi$ are infinitesimally small quantities. To the leading order, we get

$$
\begin{aligned}
& 3 p\left(1-P^{*}\right)[\delta \pi]^{2}-\left\{1-6 p\left(1-P^{*}\right) P^{*}\right\} \delta \pi \\
& -3 p\left(1-P^{*}\right)\left(P^{*}\right)^{2} \delta x=0 \quad(z=4, m=3)
\end{aligned}
$$

The reason why we have included the second order term in $\delta \pi$ in the above equation is that the coefficient of the linear term in $\delta \pi$ vanishes at the percolation transition point $p=p_{c}, P^{*}=P_{d i s c}^{*}$. Thus, at the transition point $\delta \pi$ has a square root singularity in $\delta x$, i.e.

$$
\delta \pi(x)=P_{d i s c}^{*}(x-1)^{\frac{1}{2}}, \quad x \rightarrow 1,
$$

. It follows from equation $(11)$ that $\Pi(x)$ also has a square root singularity at $x=1$.

$$
\Pi(x)=\Pi(x=1)+C_{\Pi}(x-1)^{\frac{1}{2}}, \quad x \rightarrow 1
$$


where $C_{\Pi}$ is a constant. Using equation (11) and Stirling's formula, we get

$$
\Pi_{a}=C_{\Pi} \frac{1}{a !} \frac{(2 a-3) ! !}{2^{a}}=C_{\Pi} \frac{(2 a) !}{a 2^{2 a} a !^{2}} \sim \frac{1}{a^{\frac{3}{2}}} ; a \rightarrow \infty, p=p_{c}
$$

Equations (16), (17), and (18) hold at the bootstrap transition point $p=p_{c}$. If $p=p_{c}+\delta p$ where $\delta p$ is infinitesimally small, the coefficient of the linear term in equation (15) becomes of the order of $(\delta p)^{\frac{1}{2}}$ in view of equation (13). In this case $\delta \pi$ is determined by a quadratic equation of the form,

$$
(\delta \pi)^{2}+b(\delta p)^{\frac{1}{2}} \delta \pi-c^{2} \delta x=0
$$

where b, and $\mathrm{c}$ are constants. To the lowest order in $\delta p$ and $\delta x, \delta \pi$ is given by

$$
\delta \pi=\frac{1}{2}\left[\left\{b^{2}(\delta p)+4 c^{2} \delta x\right\}^{\frac{1}{2}}-b(\delta p)^{\frac{1}{2}}\right]
$$

Thus $\pi(x)$, and therefore $\Pi(x)$ has leading square root singularity at $x=1-\frac{b^{2}(\delta p)}{4 c^{2}}$. Consequently, $\Pi_{a}$ scales as,

$$
\Pi_{a} \sim \frac{1}{a^{\frac{3}{2}}}\left[1+\frac{b^{2}\left(p-p_{c}\right)}{4 c^{2}}\right]^{-a} ; \quad a \rightarrow \infty, p \rightarrow p_{c}
$$

We note that the power-law distribution of avalanches at $p=p_{c}^{+}$results from the fact that the coefficient of the linear term $\delta \pi$ in equation (15) vanishes at $p=p_{c}$. The coefficient of $\delta \pi$ vanishes because the equation determining $P^{*}$ has a double root at $p=p_{c}$. This property is not incidental to the case $z=4$ and $m=3$. It holds for all values of $z \geq 4$, and all values of $m$ in the range $z \geq m \geq 3$. In experiments as well as computer simulations of the model, one measures the integrated probability distribution of avalanches $\Pi_{a}^{\text {int }}$, say an avalanche of size- $a$ in a range $p=p_{1}$ to $p=p_{2}$. If we are far from the transition point, the probability of large avalanches is exponentially small. Thus the integrated probability of large avalanches is mainly determined by the fact whether the interval of integration includes the transition point or not. In case the interval contains the transition point, only a small region of of width $\frac{c^{2}}{b^{2} a}$ contributes significantly to the integral for large $a$, giving us

$$
\Pi_{a}^{i n t} \sim a^{-\frac{5}{2}}, \quad a \rightarrow \infty
$$

\section{ONE-SHOT VS. SEQUENTIAL BOOTSTRAP}

The one-shot bootstrap process yields the percolation probability $\mathrm{P}(p)$ for a random initial configuration when each site is independently occupied with probability $p$. These configurations are almost always unstable under the $m$-bootstrap process, and the number of unstable sites in them is large. Therefore the size of the avalanche that reduces the initial configuration to a stable $m$-core in the one-shot bootstrap process is large. In contrast to this, the sequential bootstrap starts with a fully occupied lattice and reduces it to an empty lattice by a series of attacks and avalanches. The avalanches in sequential bootstrap connect two nearby stable $m$-cores. We have proved in the previous section that an avalanche caused by the removal of a site in a stable $m$-core is exponentially small unless the lattice is at its critical point. Thus the sequential percolation probability $P^{s e q}(p)$ decreases linearly with lattice occupation probability $p$. As there are no loops on the Bethe lattice, any stable $m$-core is necessarily infinite, and the probability $p$ that a site is occupied is identical with the probability $P^{s e q}(p)$ that it lies on an infinite cluster.

In order to illustrate the connection between one-shot and sequential bootstrap we obtain the one-shot percolation probability $\mathrm{P}(p)$ from the data of the sequential process. The key point linking the two processes is the following. Imagine we start with a fully occupied lattice, attack a fraction $p$ of the occupied sites sequentially (without accompanying avalanches) and then subject the lattice to a bootstrap culling procedure. This will of course give the same result as the one-shot process. The sequential process allows avalanches between successive attacks, so several sites that would have been attacked in the hypothetical situation imagined above are not available to be attacked because they have been culled in avalanches. Let us call such incidences false attacks. In order to get the one-shot result from the sequential process, we should keep track of the number $k$ actual attacks in the sequential process, as well as the number of false attacks up to the $k$-th attack. When the fraction of actual attacks plus the fraction of false attacks equals $p$ the sequential percolation probability $P^{s e q}(p)$ would equal the percolation probability $\mathrm{P}(p)$ under the one-shot process. This is shown in Figure- 1 for $m=2$ (second order transition) and $m=3$ (first order transition) on a random graph with $z=4$. 


\section{AVALANCHE DISTRIBUTIONS AND ORDER OF TRANSITION}

We have shown that the cumulative probability of a large avalanche of size $a$ on a random graph decays as $a^{-5 / 2}$ in the case of 1 st as well as 2 nd order percolation transitions. In Figure- 2 we have plotted the cumulative probability distribution $\mathrm{C}(a)$ vs. $a$ on a 4-coordinated random graph for $m=2$ and $m=3$. In order to obtain good quality data in reasonable computer time (one day), we performed the simulations on a graph of $10^{4}$ nodes; the data was averaged over $10^{2}$ independent assignments of nearest neighbors for each node (wirings), and $10^{3}$ runs of the sequential bootstrap process for each wiring. Thus the average is taken over $10^{5}$ samples of a $10^{4}$-node network. Probability is calculated as probability per node. Hence $\mathrm{C}(a)$ has a range from $10^{-9}$ to 1 . The avalanche is normalized by dividing with the total number of nodes so that $a$ varies from $10^{-4}$ to 1 .

In the case of a 1st order transition, Figure-2 shows that avalanches fall into two disjointed categories. A category of extensive avalanches that are of the order of the size of the network (1st order jump discontinuity), and a category of microscopic avalanches preceding the 1st order jump. The microscopic avalanches show a remarkable $a^{-5 / 2}$ power law over several decades even in a relatively small network of $10^{4}$ nodes. In contrast to this, the power law behavior of $\mathrm{C}(a)$ in the case of the 2 nd order transition is rather difficult to see because it is masked by strong cutoff effects. At first sight, this is surprising because our analysis predicts a power-law behavior at 1st as well as 2nd order transition. It is common to see power-law behavior at a 2nd order transition rather than a 1st order transition. The present simulations appear to show the opposite of what we commonly encounter. This unexpected behavior is not confined to random graphs, but is also seen on periodic lattices and small word networks [5]. Indeed, it may be used to identify a 1st order transition that is otherwise more difficult to decide numerically [6].

The power-law distribution of avalanches at the percolation point applies in the thermodynamic limit. A finite system will always have a cutoff. Therefore the question is why the cutoff is so much shorter in the case of a 2 nd order transition? In other words, why the largest avalanche at a 2nd order transition is much shorter than the largest avalanche at a 1st order transition? The answer is relatively easy on a random graph, and it may apply to other lattices as well. On a random graph or equivalently on a Bethe lattice, finite (non-spanning) $m$-cores are unstable under bootstrap dynamics for $m \geq 2$. An occupied site must necessarily belong to a spanning cluster. However, the number of sites in a spanning cluster may vary over a very wide range covering all the sites on the lattice to a relatively small number of sites that suffice to make a spanning path through the system. For example, on a Cayley tree of $n$ generations, the total number of sites is of the order of $N \approx(z-1)^{n}$, but it takes only $2 n$ steps to go from one point on the surface to another point on the surface through the root of the tree. At the 2nd order percolation transition the system will fluctuate between an empty lattice and one having approximately $\log _{z-1} N$ occupied sites. In contrast to this, at a 1st order transition the system will fluctuate between an empty lattice and one containing occupied sites of the order of $N$. This may explain why the cutoff scales as $N$ at a 1 st order transition, and as $\log _{z-1} N$ at a second order transition. Largest avalanches scaling as $N$ at 1st order transition and $\log _{z-1} N$ at 2nd order transition are also observed on hypercubic, bcc, and triangular lattices as well as small world networks. This suggests that finite $m$-cores on periodic lattices may not contribute to an increase in the cutoff of the scale of avalanches. This is also indicated by the data shown in Figure-2 for the case $m=1$; there are finite clusters in a stable 1-core on a Bethe lattice, but its avalanche distribution is characterized by a shorter cutoff in comparison with the case $m=2$. However, more work and thought is needed to fully understand these aspects of the problem.

[1] J Chalupa, P L Leath and G R Reich, J Phys C: Solid State Phys 12, L31 (1979).

[2] M E Fisher and J W Essam, J Math Phys 2, 609 (1961).

[3] S S Manna, Physica A 261, 351 (1998).

[4] C Farrow, P M Duxbury, and C F Moukarzel, Phys Rev E 72, 066109 (2005).

[5] C L Farrow, P Shukla, and P M Duxbury, J Phys A: Math Theor 40, F581 (2007).

[6] G Parisi and T Rizzo Cond-mat 609777 (2006).

[7] D Dhar, P Shukla, J P Sethna, J Phys A 30, 5259 (1977).

[8] S Sabhapandit, P Shukla, and D Dhar, J Stat Phys 98, 103 (2000). 


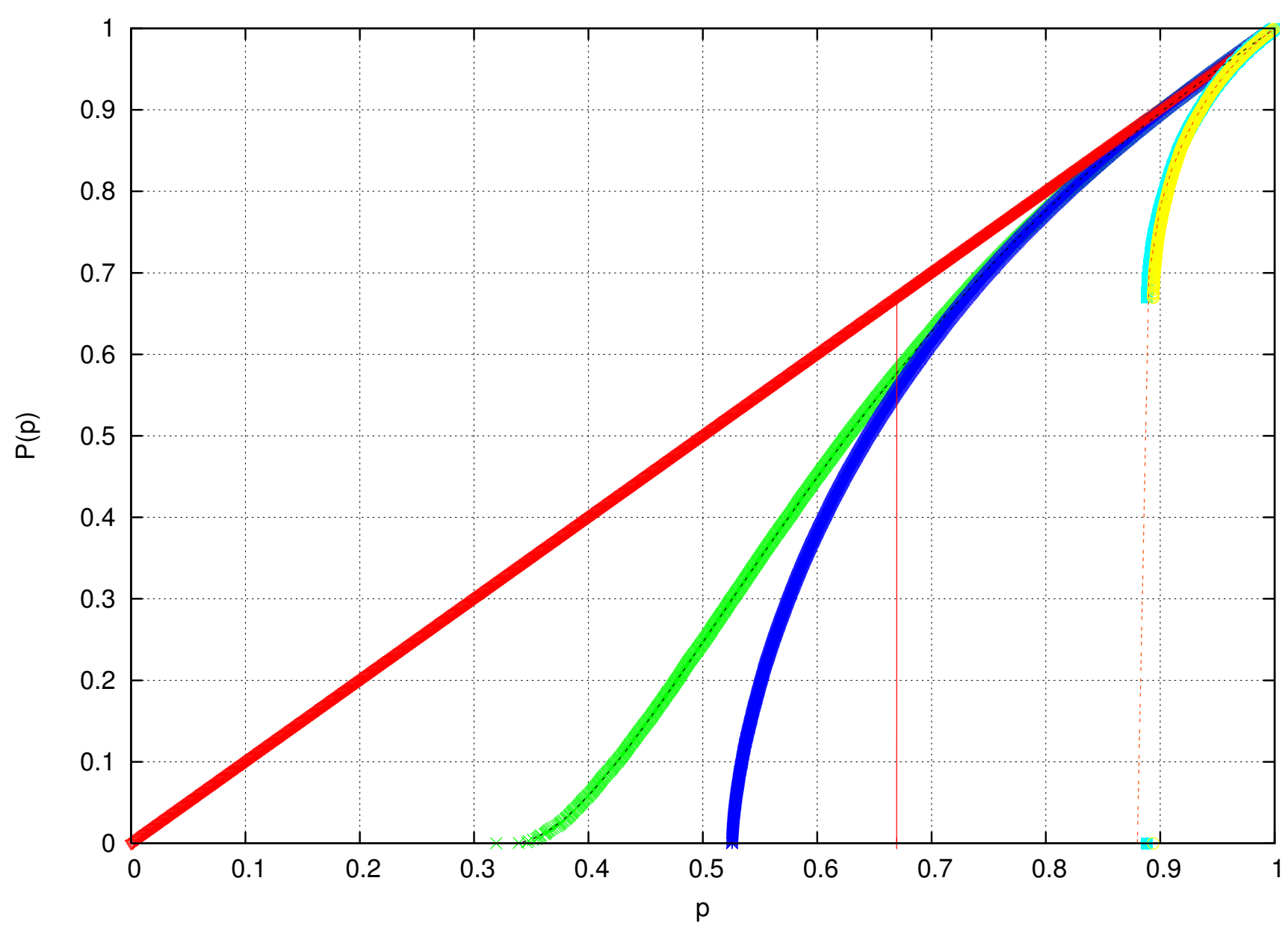

FIG. 1: The probability $\mathrm{P}(p)$ that a site on a 4-coordinated random graph belongs to a spanning $m$-core under sequential bootstrap dynamics. Curves should be viewed starting from the top right corner (fully occupied lattice). The $\mathrm{x}$-axis has a different meaning for each curve. Two nearly straight and overlapping lines that terminate at $p \approx .66$ and $p \approx 0$ respectively correspond to 1st $(m=3)$ and 2nd $(m=2)$ order transitions: in their case the x-axis denotes fractional occupation of the input lattice. The lines are nearly straight because the avalanches along them are exponentially small. The remaining curves are based the same data but plotted against different variables. For the two curves in the middle that terminate at $p \approx .52$ and $p \approx .33$; as well as the two short curves on the right that terminate at $p \approx .88$ and $P(p) \approx .65$; the x-axis denotes the $1-f_{a}$ and $1-f_{t}$ respectively where $f_{a}$ is the fractional number of sequential attacks, and $f_{t}$ is the fractional number of total attacks including false attacks (see text). The two curves in the middle are for $m=2$, and on the right for $m=3$. The curves terminating at $p=.33$ and $p=.88$ are identical with one-shot bootstrap percolation probability $P(p)$ for $m=2$ and $m=3$ respectively, as shown by the corresponding theoretical result superimposed upon them. 


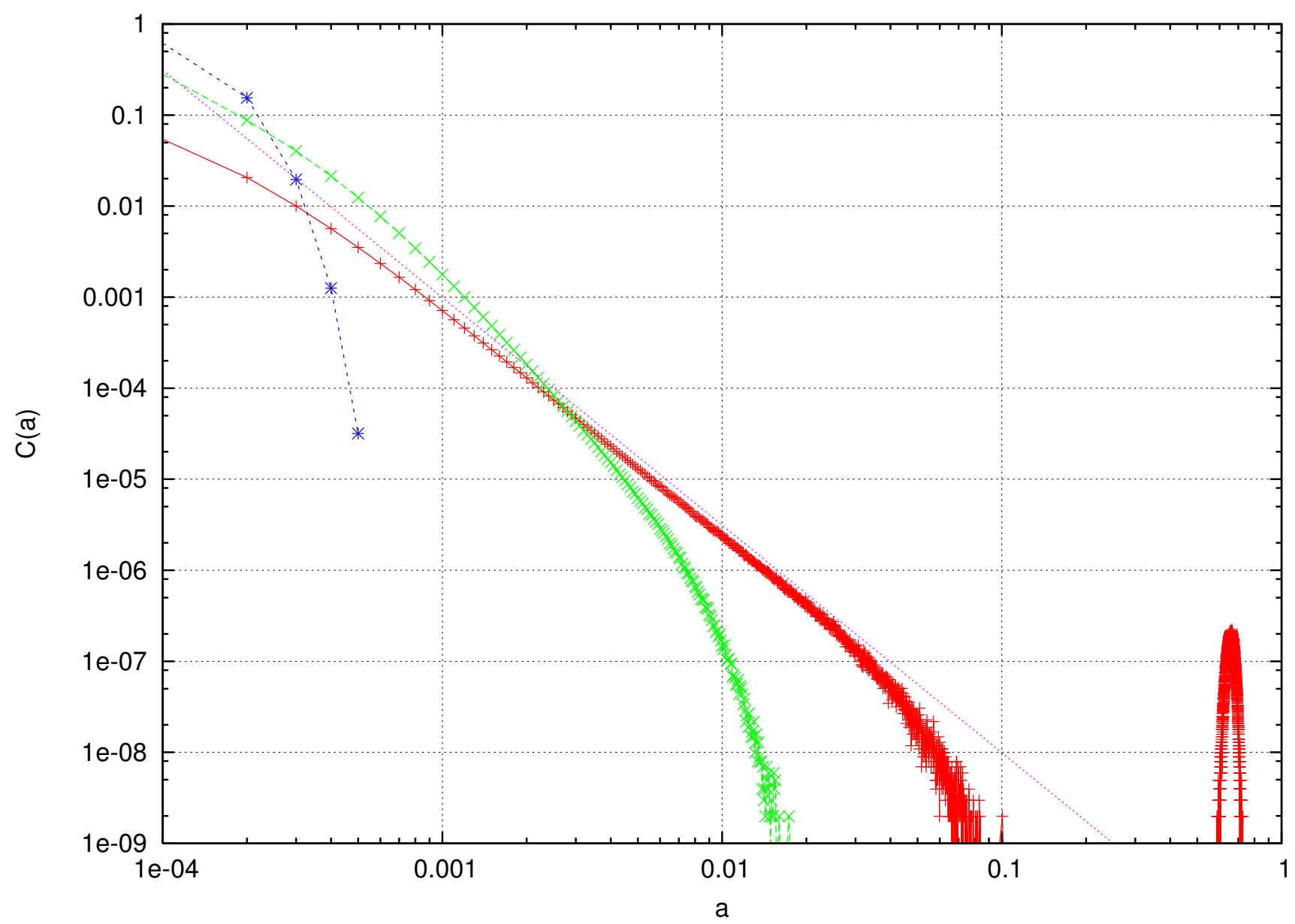

FIG. 2: Distribution of cumulative avalanches in a sequential bootstrap process for $m=1$ (blue curve), $m=2$ (green curve), and $m=3$ (red curve). The cases $m=1$ and $m=2$ correspond to continuous transitions; these cases show truncated avalanches. A discontinuous transition $(m=3)$ shows a striking power-law over several decades. A line with slope $-5 / 2$ is shown for comparison. This effect is seen in periodic lattices as well (see text). In addition, avalanche distribution in a discontinuous transition is characterized by an isolated sharp peak corresponding to extensive avalanches that empty the lattice. 